\title{
Transverse beam instabilities in the presence of linear coupling in the Large Hadron Collider
}

\author{
L. R. Carver, ${ }^{1, *}$ X. Buffat, ${ }^{1}$ K. Li, ${ }^{1}$ E. Métral, ${ }^{1}$ and M. Schenk ${ }^{1,2}$ \\ CERN, CH-1211 Geneva, Switzerland \\ EPFL, CH-1015 Lausanne, Switzerland
}

(Received 23 October 2017; published 30 April 2018)

\begin{abstract}
In the past, transverse coherent instabilities have been observed at the Hadron-Electron Ring Accelerator (HERA) proton ring that were instigated by the presence of linear coupling. Linear coupling can also potentially explain some transverse instabilities that were observed in the Large Hadron Collider (LHC) in both run I and run II, however a detailed description of the destabilizing mechanism of linear coupling was not known at the time. A study into the effect of linear coupling on transverse beam stability was carried out, and a new mechanism that could incite transverse instabilities by causing a loss of Landau damping has been found. The study includes time domain simulations with PYHEADTAIL and frequency domain computations based on analytical approaches, and was then verified by measurements with a single proton bunch in the LHC.
\end{abstract}

DOI: 10.1103/PhysRevAccelBeams.21.044401

\section{INTRODUCTION}

In run I of the LHC, many transverse coherent instabilities were observed at high energy ( 3.5 and $4 \mathrm{TeV})$ at the end of the betatron squeeze that were not fully explained [1]. The instabilities had characteristics that were consistent with the predictions from single bunch simulations, except that the Landau octupole (LO) current required to stabilize was a factor of approximately 5 higher than predicted. It was clear that a loss of Landau damping was occurring due to a mechanism whose source was not known. Linear coupling was considered as a potential cause for these instabilities, however other mechanisms were explored which allowed physics operation but ultimately were not able to fully explain the instability. In 2015, during run II of the LHC, instabilities were observed at injection energy $(450 \mathrm{GeV})$ while the machine was being filled with around 2000 proton bunches for physics operation [2]. During this process the horizontal and vertical tunes were drifting closer together due to the Laslett tune shift [3], and once the tune separation became too small emittance blowup was occurring. This instability was strongly suspected to be related to the presence of linear coupling due to its occurrence when the tune separation became small.

A headtail instability was also observed at the HERA proton ring at the beginning of the acceleration ramp [4]

*Lee.Robert.Carver@cern.ch

Published by the American Physical Society under the terms of the Creative Commons Attribution 4.0 International license. Further distribution of this work must maintain attribution to the author(s) and the published article's title, journal citation, and DOI. where it was measured that linear coupling was an essential ingredient for the instability to occur. An explanation was put forward in the framework of "coupled Landau damping" [5], where the possibility that linear coupling could cause a loss of Landau damping was discussed. However, only a simple analytical model with an externally given tune spread was proposed and the exact mechanism for the impact of linear coupling on the Landau damping was not developed.

These observations are seemingly in contradiction to measurements that were made in the CERN Proton Synchrotron (PS) [6,7]. In the PS, a horizontal headtail instability was observed at injection energy $(1.4 \mathrm{GeV})$ that was able to be cured by the introduction of linear coupling. The linear coupling in this case was shown to be beneficial due to the sharing of the instability rise times in each plane, allowing stabilization of the horizontal plane through coupling to the stable vertical plane. This was performed in the absence of both octupoles and a transverse feedback. Simulation studies were also performed in the SPS that also showed a beneficial effect of linear coupling by increasing the threshold for the transverse mode-coupling instability [8] in one plane by coupling to the other.

It is clear that linear coupling can strongly impact the transverse beam stability, but the exact mechanism of this interaction has not been studied in detail. It is well known that the introduction of linear coupling changes the coherent motion of the beam as it moves around the accelerator [9]. This can complicate the way nonlinear elements interact with the beam. In the specific case of the LHC, the effect of linear coupling on nonlinear observables [such as residual amplitude detuning from the 
interaction regions (IRs)] has been extensively studied [10-12] and shown to have a large impact. A more general model for how linear coupling interacts with the LO's that can be applied to any machine has been derived by $\mathrm{R}$. Tomás et al. [13]. However in all of these studies, the link between the optics parameters and beam stability has not been made.

This paper will present the results of a series of analytic and simulation studies that aim to show how linear coupling can affect the single bunch transverse stability by modification of the amplitude detuning from the LOs. Time domain simulations will be performed using PYHEADTAIL [14-16], a self-consistent 6D macroparticle tracker. These will be compared with an analytic approach which consists of several frequency domain calculations. These calculations start with an estimation of the transverse stability threshold based on the coherent tune shifts obtained from the frequency domain Vlasov solver DELPHI [17] and is then followed by a numerical estimation of the dispersion integral using the PYSSD (Python Solver for Stability Diagrams [18]) code. PYSSD requires as input the transverse amplitude detuning due to the lattice which is computed with single particle tracking using MAD-X [19].

The results of this simulation study were able to be tested with controlled measurements with a single bunch in the LHC at an energy of $6.5 \mathrm{TeV}$. Some examples when uncontrolled instabilities occurred during physics operation, which have since been attributed to the destabilizing mechanism caused by linear coupling, will be briefly mentioned. Chronologically speaking, the simulation model was developed before the controlled measurement was carried out and the full numerical analysis came afterwards. Here the results will be described in a more logical progression.

Section II will introduce some of the basic relevant physics behind linear coupling in an accelerator system. Then the analytic results computed using frequency domain techniques will be shown in Sec. II. The time domain simulations will then be introduced and described in Sec. III. Controlled instability measurements will be described in Sec. IV, before an application of the results found here will be applied to the LHC stability model which is shown in Sec. V.

\section{LANDAU DAMPING IN THE PRESENCE OF LINEAR COUPLING}

\section{A. Basic concepts}

To model linear coupling appropriately, some consistent definitions need to be introduced [20]. First, to define the strength of the coupling resonance the parameter $\left|C^{-}\right|$is used, which is the minimum tune separation achievable in the coupled system. This parameter is also called the closest tune approach and is a global property of the full lattice and can be easily obtained by performing a tune crossing, where a clear separation between the measured fractional tunes in the horizontal and vertical spectra will be observed. However if the tune separation is large compared to the $\left|C^{-}\right|$, then the effect of coupling on the transverse motion will be small.

The tune separation is defined as $Q_{\text {sep }}=\left|Q_{y}-Q_{x}\right|$, where $Q_{x}$ and $Q_{y}$ are the uncoupled horizontal and vertical fractional tunes. When coupling is present a tune shift occurs, and the new coupled tune separation is defined as $Q_{\text {sep,coupled }}=\left|Q_{v}-Q_{u}\right|$ where $Q_{u}$ and $Q_{v}$ are the peaks observed in the horizontal and vertical spectra. The tune shift that is experienced is given by Eqs. (1) and (2) [21]

$$
\begin{gathered}
Q_{u}-Q_{x}=-\frac{1}{2}\left(-Q_{\mathrm{sep}}+\sqrt{Q_{\mathrm{sep}}^{2}+\left|C^{-}\right|^{2}}\right) \\
Q_{v}-Q_{y}=\frac{1}{2}\left(-Q_{\mathrm{sep}}+\sqrt{Q_{\mathrm{sep}}^{2}+\left|C^{-}\right|^{2}}\right) .
\end{gathered}
$$

In the LHC a tune feedback is used to ensure that the measured coupled horizontal and vertical tunes remain at the desired value. At $6.5 \mathrm{TeV}$ and during collision, the desired fractional tunes are $Q_{x}=0.31, Q_{y}=0.32$. When linear coupling is present, the measured tunes are not $Q_{x}$, $Q_{y}$ but $Q_{u}, Q_{v}$. Therefore the tune feedback is keeping the coupled tunes at their desired values. This intrinsically increases the effect of linear coupling on the system. In the event that the $\left|C^{-}\right|$increases, the tunes begin to shift away from the coupling resonance. With the feedback on, tune trims are applied to ensure that the tunes remain constant, which reduces the uncoupled separation $Q_{\text {sep }}$ and amplifies the effect of linear coupling on the beam dynamics.

A coupling feedback is also in development in the LHC $[22,23]$. When operational this would provide fast and accurate coupling measurements throughout the dynamic phases of LHC operation (for example the energy ramp or the betatron squeeze). This information will then be used to apply trims to the skew quadrupole corrector magnets, hence reducing the overall coupling in the machine. A coupling feedback would allow reliable operation at very small tune separations and many of the problems that will be described here would not occur.

\section{B. Effect of linear coupling on Landau octupoles}

In the LHC, the required betatron tune spread to stabilize the transverse coherent instabilities through Landau damping is provided by dedicated Landau octupoles (LOs) [24]. An unstable mode has a real and imaginary tune shift, (the real part is seen as a physical tune shift, while the imaginary denotes an instability rise time). The ability for the tune spread to damp this mode is determined by the stability diagram theory $[25,26]$, but one can use a simple model. If the real part of the unstable mode lies within the projection of the tune spread on both the horizontal and vertical axes, 


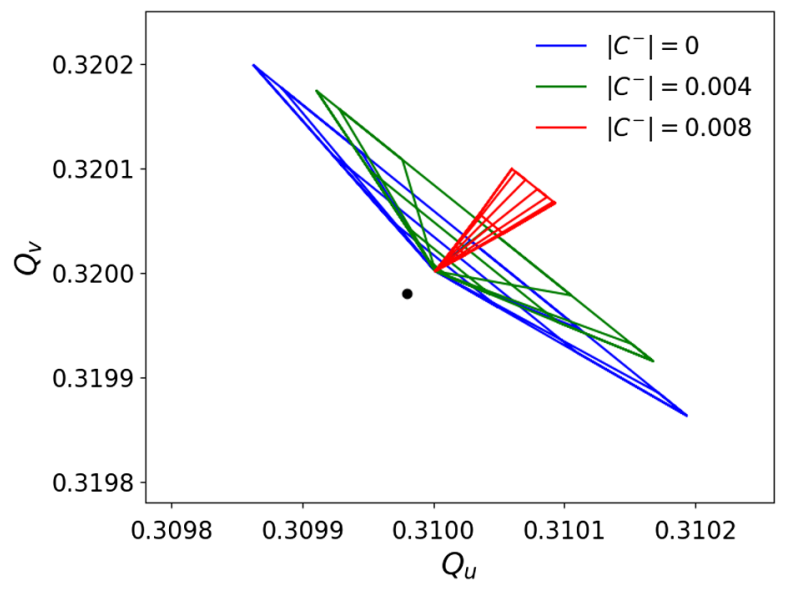

FIG. 1. MAD-X tracking results showing the tune footprint for different values of $\left|C^{-}\right|$and constant tunes with $J_{\text {oct }}=500 \mathrm{~A}$. The particle amplitude is extended to $4 \sigma$. A clear impact on the tune spread can be observed as the coupling approaches the coupled tune separation. This severely inhibits the ability of the LOs to stabilize against a typical unstable mode (black point).

then the mode can potentially be stabilized (depending on the imaginary part). If the mode lies outside of this spread, then it cannot be stabilized through Landau damping.

The effect of linear coupling on the tune spread and consequently on the Landau damping can be studied using the MAD-X tracking code. To start with, the LHC lattice model of 2016 is used and a single skew quadrupole is placed at the center of Interaction Point 3 (IP3). This single skew quadrupole can be powered to provide linear coupling throughout the lattice. In this case, there is no local variation of coupling throughout the ring, and the $\left|C^{-}\right|$can be used to quantify the strength of the linear coupling. Calibration studies were performed which allowed to determine the relationship between the strength of the skew quadrupole and the $\left|C^{-}\right|$. The LOs were powered with $J_{\text {oct }}=500 \mathrm{~A}$, and the tunes were kept constant at $Q_{u}=0.31, Q_{v}=0.32$. Single particles initialized at different amplitudes are then tracked through the lattice for 1024 turns and the tune of each of the particles is determined with a high accuracy through an interpolated Fourier transform [27]. The amplitude dependent tune $Q_{u, v}\left(J_{u}, J_{v}\right)$ is thus obtained numerically and it is conveniently represented as a tune footprint, where the tune of each particle oscillating at different amplitude is drawn on the tune diagram. The effect of the linear coupling on the tune spread generated by the LOs is illustrated in Fig. 1. In order to possibly Landau damp an unstable mode, the real part of the coherent shift must lie within the projection of the footprint on both the horizontal and vertical axes. Linear coupling has a clear impact on the amplitude detuning generated by the LOs, which can lead to a loss of Landau damping. This can be seen by the fact that for no coupling or small coupling (blue and green curves), the black point lies within the projection of the footprint, while for strong coupling (red footprint) the tune spread is severely diminished.

Throughout this study, the magnitude of the tune spread will be described in units of Amperes (relating to the amount of current given to the LOs). It is important to explain how the current in the LOs relates to the tune spread. Typically, the amount of detuning provide by LOs is given by [28]

$$
\begin{aligned}
& \Delta Q_{x}\left(J_{x}, J_{y}\right)=\alpha_{x x} J_{x}+\alpha_{x y} J_{y} \\
& \Delta Q_{y}\left(J_{x}, J_{y}\right)=\alpha_{y y} J_{y}+\alpha_{y x} J_{x},
\end{aligned}
$$

where $J_{x}$ and $J_{y}$ are the transverse actions of the particle and $\alpha$ corresponds to the detuning coefficient. These detuning coefficients can be analytically calculated according to the strength of the octupoles and the values of optics parameters at the octupoles around the ring. In the case of the LHC at $6.5 \mathrm{TeV}$ the coefficients can be computed as [29]

$$
\begin{aligned}
& \alpha_{x x} \approx 287608 \frac{J_{\mathrm{oct}}^{F}}{550}-8460 \frac{J_{\mathrm{oct}}^{D}}{550}, \\
& \alpha_{y y} \approx 10542 \frac{J_{\mathrm{oct}}^{F}}{550}-298526 \frac{J_{\mathrm{oct}}^{D}}{550}, \\
& \alpha_{x y}=\alpha_{y x} \approx-110127 \frac{J_{\mathrm{oct}}^{F}}{550}+100510 \frac{J_{\mathrm{oct}}^{D}}{550},
\end{aligned}
$$

where $J_{\text {oct }}^{F}$ and $J_{\text {oct }}^{D}$ refers to the octupole current at the LOs placed alongside a focusing quadrupole and defocusing quadrupole, respectively.

A description on how to make this calculation can be found in Refs. [28,29]. A fundamental description of how linear coupling can cause this effect on the amplitude detuning can be found in Ref. [13]. The interplay between the non-linear fields of the octupole and skew quadrupolar errors can be described following the Hamiltonian approach. Particle tracking simulations are preferred when modeling this interplay as practical analytic solutions do not exist for arbitrary cases.

The appendix shows the results of a study that compares the effect of local linear coupling and global linear coupling. The appendix shows that what contributes to the distortion of the tune footprint from the LOs is the magnitude of the linear coupling resonance driving terms (RDTs) at the LOs rather than the global coupling given by the $\left|C^{-}\right|$. However, in the regime where only one skew quadrupole is used, or that the coupling is large (which means that the global effects dominate over local variations), local and global coupling are directly related.

\section{Stability theory in presence of linear coupling}

The Discrete Expansion over Laguerre Polynomials and Headtail Modes to compute Instabilities (DELPHI) code is 
a frequency domain Vlasov solver that takes a machine impedance and bunch parameters as input, and calculates all the complex coherent modes that can develop [17]. It can also include first order chromaticity and transverse feedbacks in the computation of the complex mode frequencies. From the unstable modes it is straightforward to determine what is the most critical mode and the tune spread that is required in order to stabilize it. However, DELPHI is not yet able to model linear coupling directly, so a slightly different approach is required. Firstly, it is assumed that both planes are approximately the same (including impedance, chromaticity and transverse feedback). Linear coupling causes a modification of many different beam dynamics effects, but one of the effects that linear coupling has on the impact of LOs is a rotation. The transformation from $Q_{x}, Q_{y}$ to $Q_{u}, Q_{v}$ can be modelled as an effective rotation, which reduces the effect of normal octupoles and increases the effect of skew octupoles. This is what changes the detuning coefficients of the tune footprints shown in Fig. 1. In the case of the complex coherent modes, if both planes are similar then the unstable mode that develops in the uncoupled frame has the same characteristics as the mode that develops in the coupled frame (as the rotation will result in coupled modes of the same real and imaginary magnitudes). This allows an uncoupled treatment to be applied to the coupled unstable modes to determine their stability in the presence of a tune spread.

In order to check if this is valid for the case of the LHC, the ratio between the horizontal and vertical impedances can be plotted in the frequency range of interest. This frequency range is determined by the convolution of the bunch modes, which for a Gaussian beam and no chromaticity is given by Eq. (5) [3], and the full absolute machine impedance. This gives an effective impedance, which is the part of the impedance that contributes to the unstable mode.

$$
h_{\ell}(\omega)=\left(\frac{\omega \sigma_{z}}{c}\right)^{2 \ell} e^{-\omega^{2} \sigma_{z}^{2} / c^{2}},
$$

where $\sigma_{z}$ is the one-sigma bunch length in the z-coordinate, $c$ is the velocity of light, $\ell$ is the mode number for the bunch decomposition and $\omega$ is the angular frequency. When chromaticity is introduced, the bunch modes are shifted by the chromatic frequency

$$
\omega_{\xi}=\frac{\xi \omega_{\beta}}{\eta}
$$

where $\xi=\frac{\Delta Q / Q}{\Delta p / p}$ is the first order chromaticity, $\omega_{\beta}=\omega_{0} Q$, $\omega_{0}$ is the revolution angular frequency, $Q$ is the tune (integer and fractional) and $\eta$ is the slip factor. In the LHC, the terminology used to denote the chromaticity is $Q^{\prime}$ where $Q^{\prime}=Q \xi=\frac{\Delta Q}{\Delta p / p}$. Typical values used in operation range between $Q^{\prime}=0$ and $Q^{\prime}=20$.

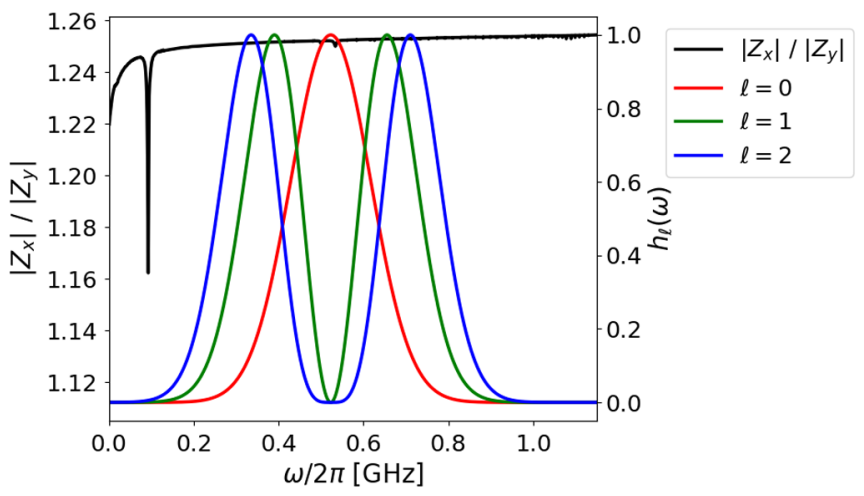

FIG. 2. Ratio of the horizontal to vertical impedances (black) of the LHC at 6.5 TeV plotted alongside the first three bunch modes (red, green and blue).

By making the substitution $\omega \rightarrow \omega+\omega_{\xi}$ for $Q^{\prime}=15$ and plotting the first three bunch modes alongside the ratio of the impedance, it is clear to see that the horizontal impedance is approximately $25 \%$ larger than the vertical impedance. This is shown in Fig. 2.

The tune spread required to stabilize a coherent mode of oscillation with a given coherent tune shift with respect to the unperturbed tune $\Delta Q_{i}$ obtained by the modal analysis in a given machine and beam configuration is determined using the dispersion integral [26]

$\frac{-1}{\Delta Q_{i}}=\iint_{0}^{\infty} \frac{J_{i} \frac{d \psi}{d J_{i}}}{Q-Q_{i}\left(J_{u}, J_{v}\right)} d J_{u} d J_{v}, \quad i=u, v$,

where $\Psi\left(J_{u}, J_{v}\right)$ is the particle distribution assumed to be exponential in the transverse coupled actions $J_{u}$ and $J_{v}$, and therefore Gaussian in physical space. $Q$ is the

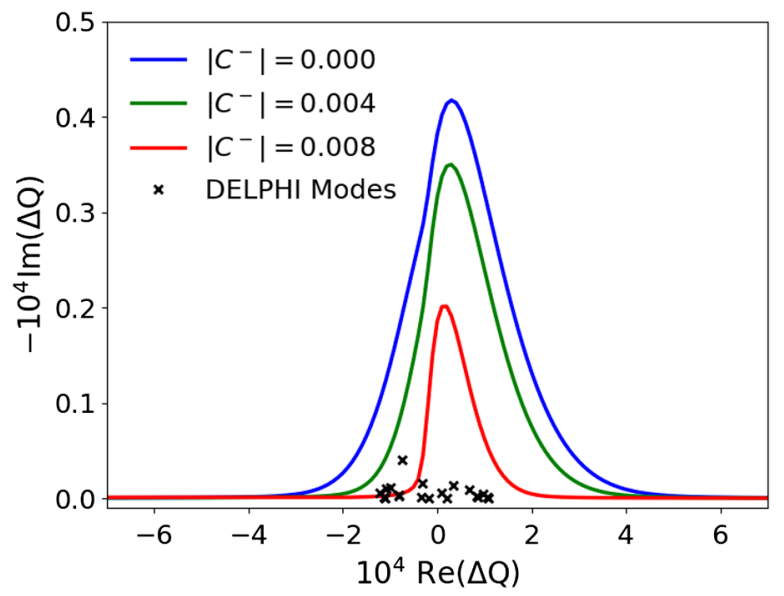

FIG. 3. Stability diagram in the complex tune space as a function of different values of $\left|C^{-}\right|$plotted alongside the complex coherent modes from DELPHI. As the coupling is increased, some previously stable modes lie outside of the stable region and Landau damping is lost. The coupled tunes are kept constant with $Q_{u}=0.31$ and $Q_{v}=0.32$. 
TABLE I. Bunch and machine parameters for a full machine in 2016 that was used to compute the stability diagram shown in Fig. 3.

\begin{tabular}{lcc}
\hline \hline Parameter & Value & Unit \\
\hline Number of Bunches $M$ & 2748 & $\ldots$ \\
Beam Energy & 6.5 & $\mathrm{TeV}$ \\
$\beta^{*}$ IP1/5 & 40 & $\mathrm{~cm}$ \\
Bunch intensity $N_{b}$ & 1.2 & $10^{11} p$ \\
Bunch normalized emittance $\varepsilon_{x}, \varepsilon_{y}$ & 2,2 & $\mu \mathrm{m}$ \\
Bunch length $4 \sigma_{t}$ & 1.2 & $\mathrm{~ns}$ \\
Transverse feedback damping time $\tau_{b}$ & 100 & turns \\
Chromaticity $Q_{x}^{\prime}, Q_{y}^{\prime}$ & 10,10 & $\cdots$ \\
\hline \hline
\end{tabular}

coherent resulting tune one is looking for taking into account the effect of the tune spread, modelled as a perturbation. Thus, by varying $Q$ on the real axis, the contour defines the boundary in the complex plane where an oscillation mode with a coherent tune shift $\Delta Q_{i}$ obtained from the linear theory, i.e. in absence of tune spread, is at the limit of stability by the presence of a given tune spread. The representation of this boundary is called a stability diagram, where stability is assumed if the unstable mode lies within the curve. By comparing the complex tune shift of all the oscillation modes obtained with the linear theory to the stability diagram, one can deduce the beam stability. In particular, a stability criterion is defined called the "coherent stability factor" that allows the beam stability to be quantified by considering the smallest relative distance of the tune shifts with respect to its projection on the stability diagram among all the coherent modes. A coherent stability factor above the unit indicates a beam instability, whereas if it is below the unit it indicates beam stability.

Figure 3 shows the effect of the modification of the tune footprint due to linear coupling shown in Fig. 1, obtained by integrating numerically Eq. (7) with the amplitude detuning derived with MAD-X. The coherent tune shift expected with the LHC machine and beam parameters of the 2016 run (shown in Table I) are marked as crosses. Some modes lose Landau damping as the strength of linear coupling is increased. This mechanism will be demonstrated with multiparticle tracking simulation and with comparison to experimental data.

\section{PyHEAdTAIL SIMULATIONS}

\section{A. Simulation setup}

PYHEADTAIL is a macroparticle tracking code designed specifically to simulate collective effects in circular machines. A linear map is created that can include kicks from a variety of different elements, including wakefields, feedback systems, magnetic elements, or arbitrary user defined kicks as well as longitudinal maps to account for synchrotron motion or amplitude detuning from

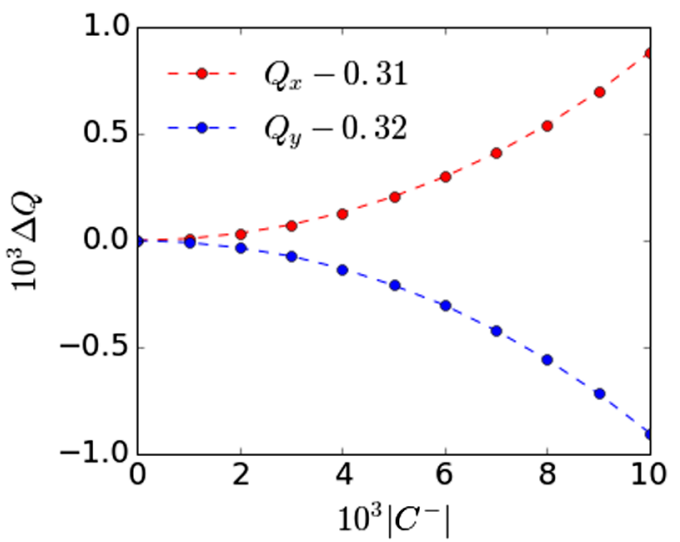

FIG. 4. Initial uncoupled horizontal (red) and vertical (blue) tune shifts required in order to maintain collision tunes.

octupoles. For the examples that will be shown here, a linear map that is comparable to a simplified LHC is constructed. This model slices the bunch longitudinally and calculates a kick from the wakefield that propagates from one slice to the next and also includes a single skew quadrupole kick in order to introduce coupling and a longitudinal map to account for chromatic and synchrotron effects.

Octupoles can also be modeled by introducing a transverse detuning that follows Eq. (4). The detuning coefficients are computed using MADX and the values are used as input in PyHEADTAIL. The transverse actions of each macroparticle is calculated individually and the relevant change is made to its phase advance in the horizontal and vertical planes to account for the amplitude detuning.

The aim of the simulations is to mimic the behaviour of the LHC, but in a reduced and simplified scenario. It was shown in Sec. II that when coupling is introduced, a tune shift occurs that can be calculated from Eqs. (1) and (2). However, in the LHC during operation the tune feedback is active. This is a system that calculates the tune in real time and applies small changes to specified corrector quadrupoles to ensure the tunes remain at the desired values. These values are either at injection tunes $\left(Q_{x}=0.28\right.$, $\left.Q_{y}=0.31\right)$ or collision tunes $\left(Q_{x}=0.31, Q_{y}=0.32\right)$, depending on the stage of the operation. In the event that coupling is introduced with the tune feedback on, the coupled tunes $Q_{u}$ and $Q_{v}$ will not shift but the uncoupled tune separation $Q_{\text {sep }}$ will become different.

In order to determine the magnitude of this tune trim, a 2D minimization function was implemented with PYHEADTAIL using only a skew quadrupole to introduce global coupling. The initial tunes were varied until they gave the desired tunes $\left(Q_{u}=0.31, Q_{v}=0.32\right)$, and this was performed as a function of the $\left|C^{-}\right|$. The results of this scan are shown in Fig. 4. In order to maintain collision tunes when the $\left|C^{-}\right|$is equal to the coupled tune separation, a shift on the order of $\approx 10^{-3}$ is needed to be applied in both planes. 
TABLE II. Bunch and machine parameters used for the PyHEADTAIL simulations. The values used were to reduce the level of complexity of the simulations.

\begin{tabular}{lcc}
\hline \hline Parameter & Value & Unit \\
\hline Number of bunches $M$ & 1 & $\ldots$ \\
Beam energy & 6.5 & $\mathrm{TeV}$ \\
$\beta^{*}$ IP1/5 & 3 & $\mathrm{~m}$ \\
Bunch intensity $N_{b}$ & 1.1 & $10^{11} p$ \\
Bunch normalized emittance $\varepsilon_{x} / \varepsilon_{y}$ & $2 / 2$ & $\mu \mathrm{m}$ \\
Bunch length $4 \sigma_{t}$ & 1.2 & $\mathrm{~ns}$ \\
Transverse feedback damping time $\tau_{b}$ & $\infty$ & turns \\
Chromaticity $Q_{x}^{\prime} / Q_{y}^{\prime}$ & $1 / 1$ & $\ldots$ \\
Number of macroparticles & 6 & $10^{5}$ \\
Number of turns simulated & 1.5 & $10^{6}$ \\
\hline \hline
\end{tabular}

Rather than try to perform these simulations with operational parameters $\left(2748\right.$ bunches with $\left(Q_{x}^{\prime}, Q_{y}^{\prime}\right)=(15,15)$, strong octupoles and a strong transverse feedback), we perform these simulations with more favorable parameters in order to determine the fundamental impact of coupling on the transverse stability. These parameters can be found in Table II.

Scans in the LO current were then performed as a function of $\left|C^{-}\right|$in order to determine how much current is required for stabilization. The tunes were able to be maintained by using the information from Fig. 4 combined with a fixed tune shift from the bunch intensity (countering the effect of the bunch intensity interacting with the impedance). The PYHEADTAIL simulation results will be compared to the results from PYSSD, as described in the following section.

\section{B. Simulation results}

As an initial test of the simulation results, the tunes were calculated for each $\left|C^{-}\right|$step with $J_{\text {oct }}=0$ A. By performing the tune analysis on the first 20000 turns, before the instability has started, the tune can be determined. The same analysis can then be repeated for the last 20000 turns and the real coherent tune shift $\Delta Q_{\text {coh }}$ from the instability can be determined. The results of these calculations can be found in Fig. 5.

The uppermost plot shows the simulated tunes vs the $\left|C^{-}\right|$. It is clear to see that the tunes are constant throughout the scan, with the horizontal tune having a constant offset of approximately $-3.3 \times 10^{-5}$ while the vertical is even closer (save for one outlier with an offset of $\approx 10^{-4}$ that is not shown. On the scale of the effects that are being studied, this offset is negligible. The lower plot shows the real part of the coherent tune shift and its evolution with the introduction of linear coupling. It can be seen that this shift varies by approximately $0.5 \%$ when introducing the maximum possible coupling (when $\left|C^{-}\right|=Q_{\text {sep,coupled }}$ ). The introduction of linear coupling does not have an impact on the real coherent tune shift.
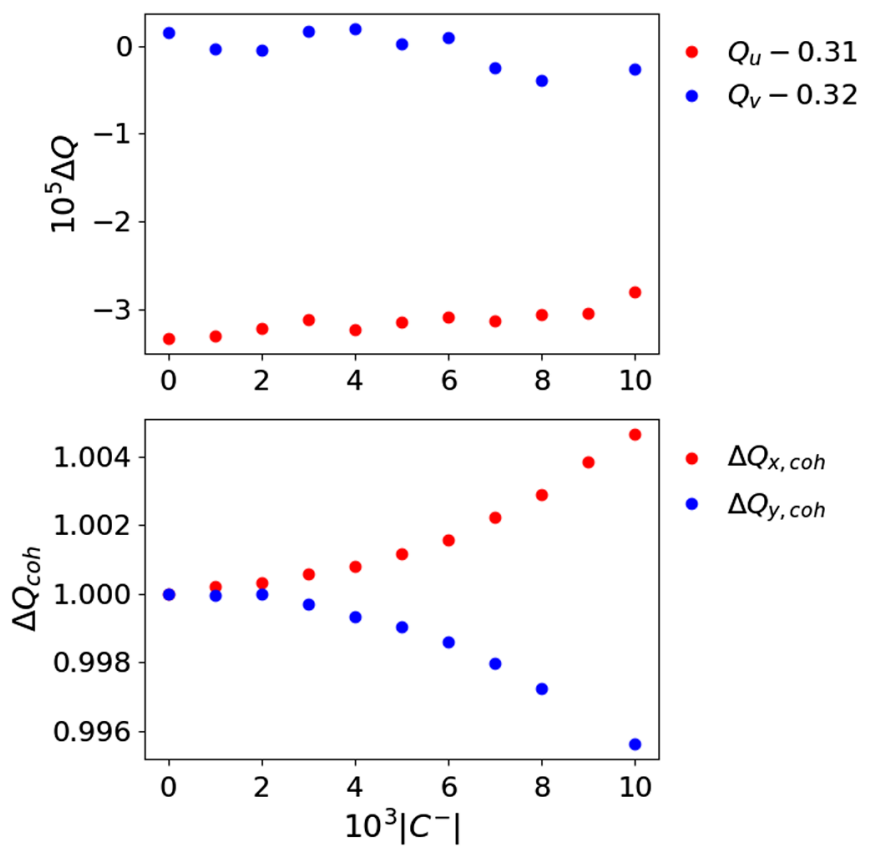

FIG. 5. Top: Simulated tunes after the introduction of linear coupling. It can be seen that all tunes are within $10^{-4}$ (one outlier not shown) of the required value. Bottom: Coherent tune shift of the instability as a function of the linear coupling. A variation on the order of $0.4 \%-0.5 \%$ can be seen for $\left|C^{-}\right|=0.01$.

In terms of the instability characteristics, due to the slightly higher impedance in the horizontal plane the dominant instability was in horizontal. Therefore the analysis will mostly contain instabilities in the horizontal plane but in some cases the horizontal instability does not allow clean calculations for the vertical plane.

Figure 6 shows the headtail mode trace of the horizontal instability (for $J_{\text {oct }}=0 \mathrm{~A}$ ) with and without linear coupling. It can be seen that the mode profile is the same in both cases, and shows a radial mode $|l|=1$. Figure 7 shows the instability rise time for $J_{\text {oct }}=0 \mathrm{~A}$ as a function of the coupling. The instability rise time is directly related to the

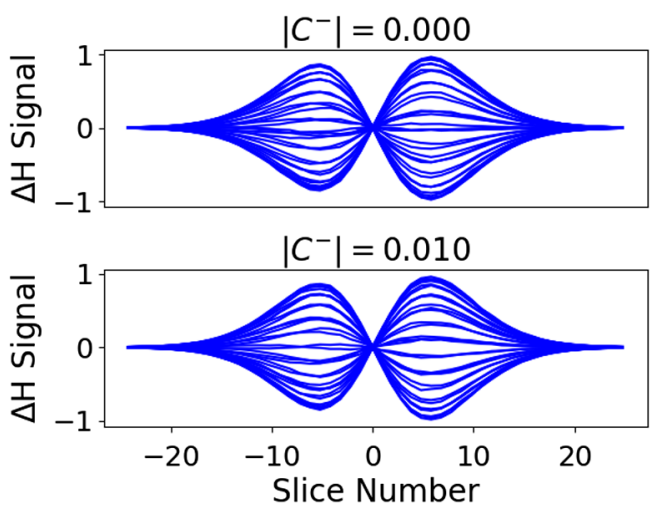

FIG. 6. Comparison of headtail pattern with $J_{\text {oct }}=0$ A for the case without coupling (top) compared to the case with coupling (bottom). 


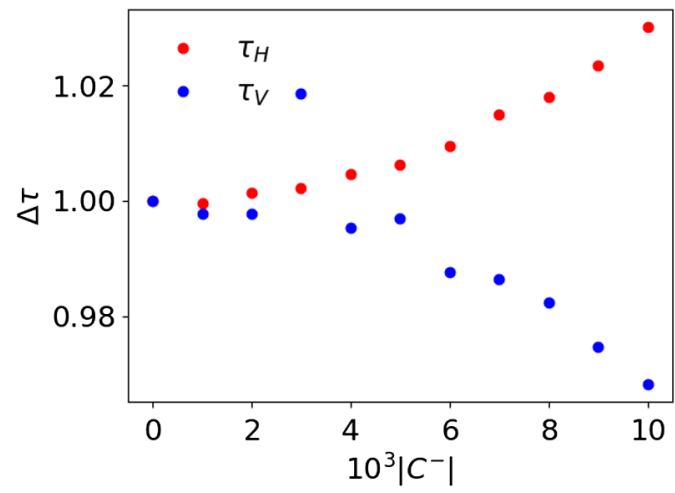

FIG. 7. The change of instability rise time with coupling. The initial starting values were $\tau_{H}=5.66 \mathrm{~s}$ and $\tau_{V}=7.65 \mathrm{~s}$.

imaginary part of the complex coherent tune shift, and it can be seen that it too has a small variation with coupling. From each of these observations (complex coherent tune shifts and headtail pattern) it can be seen that the fundamental characteristics of the instability do not change. This adds further weight to the previous assertion that the linear coupling mechanism is purely a loss of Landau damping, as well as validating the assumption that this work is applicable only in the case where the impedance, chromaticity, and transverse feedback properties are similar for both planes.

The fact that the variation of the rise time with coupling is on the order of $\approx 2 \%-3 \%$ while the variation of the real coherent tune shift with coupling is on the order of $\approx 0.3 \%$ is another validation of the measurements made in the CERN PS. Linear coupling has a much stronger effect on the instability rise time by sharing it between the planes, while having a minimal effect on the real coherent tune shift. A fully developed analytical theory will cast a light on why this is the case.

The results of the stabilizing octupole scan in PYHEADTAIL is shown alongside the results of equivalent PYSSD computations in Fig. 8. Both sets of results are plotted normalized to their uncoupled stabilizing threshold, such that the figure is showing the relative increase in the required octupole current as a function of linear coupling.

The figure shows clearly that similar effects are being observed in PyHEADTAIL as was observed in PYSSD. As the linear coupling increases, more octupole current is required to fully stabilize the single bunch against coherent instabilities. There is some small disagreement in the region $4 \times 10^{-3}<\left|C^{-}\right|<8 \times 10^{-3}$ that could arise from a number of reasons from the fact that PyHEADTAIL can only simulate a finite number of turns whereas frequency domain analysis is for infinite turns. The most striking difference between the two sets of results is at $\left|C^{-}\right|=0.01$, where PYSSD shows a large reduction in the required octupole current whereas PyHEADTAIL shows a continuation of the steady increase. This is likely due to the fact that the stability diagram theory makes the assumption that

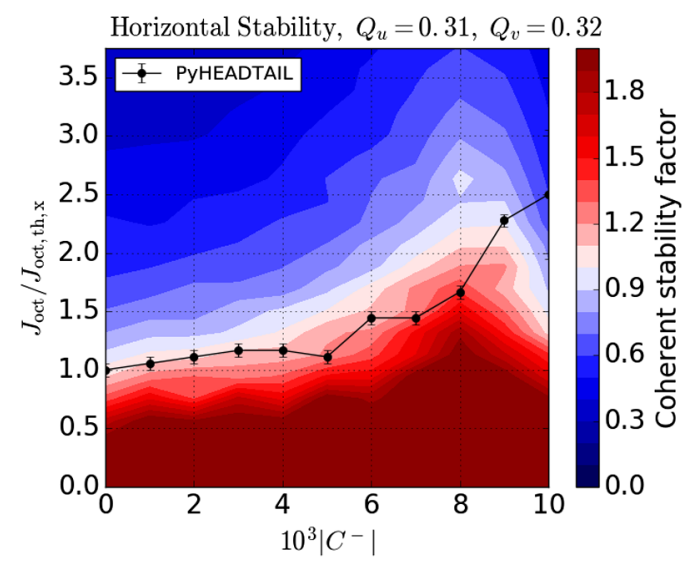

FIG. 8. Stablizing octupole current (normalized to uncoupled value) as a function of the $\mid C^{-}$for both PySSD (contours) and PyHEADTAIL. For each step the tunes are kept at constant values of $Q_{u}=0.31, Q_{v}=0.32$.

the particle dynamics are not dominated by resonances. Clearly in the strongly coupled case, this assumption breaks down. PYHEADTAIL is not limited by this assumption. At first glance it may appear that these simulation results do not agree with the simple model shown in Fig. 1. If the octupole current is increased then the mode will clearly not lie within the project of the footprint. However what the simple model does not include is the fact that for higher octupole currents, the contour of the stability diagram increases which also has the effect of increasing the rise time for the unstable modes. It is therefore possible that over the timescales simulated in the time domain, the rise times increase until an instability does not develop.

This section has shown that linear coupling can cause a loss of Landau damping through a modification of the tune footprint, without changing fundamentally any of the characteristics of the instability. The next section will show the results of some beam based measurements on transverse beam stability in the presence of linear coupling that were performed in the LHC at $6.5 \mathrm{TeV}$, as well the results of some uncontrolled instabilities in physics operation that have been linked to linear coupling.

\section{BEAM BASED MEASUREMENTS IN THE LHC}

Measurements have been made in the LHC that aim to verify the impact of linear coupling on transverse beam stability. Two sets of controlled measurements were made with a single bunch in dedicated studies. The first determined the stabilizing octupole threshold without linear coupling. The second set of measurements went to identical machine conditions, but made a single bunch become unstable through linear coupling in the presence of high octupole currents. The thresholds of each case and the instability characteristics can be compared in order to verify the simulation results from Sec. III. More information 
TABLE III. Bunch and machine parameters during the controlled stability measurements in the absence of linear coupling.

\begin{tabular}{lcc}
\hline \hline Parameter & Value & Unit \\
\hline Number of bunches $M$ & 1 & $\ldots$ \\
Beam energy & 6.5 & $\mathrm{TeV}$ \\
$\beta^{*}$ IP1/5 & 3 & $\mathrm{~m}$ \\
Bunch intensity $N_{b}$ & 1.2 & $10^{11} p$ \\
Bunch normalized emittance $\varepsilon_{x}, \varepsilon_{y}$ & $1.5,1.75$ & $\mu \mathrm{m}$ \\
Transverse feedback damping time $\tau_{b}$ & 100 & turns \\
Chromaticity $Q_{x}^{\prime}, Q_{y}^{\prime}$ & 9,8 & $\cdots$ \\
\hline \hline
\end{tabular}

about the measurements will be provided in the next section.

\section{A. Controlled measurements}

In 2016 two instability measurements were performed in the $\mathrm{LHC}$ at $6.5 \mathrm{TeV}[2,30]$. For each measurement, a single bunch with intensity $N_{b} \approx 1.1 \times 10^{11} \mathrm{p}$ and normalized emittance $\left(\varepsilon_{x}, \varepsilon_{y}\right) \approx(2,2) \mu \mathrm{m}$ was accelerated to 6.5 TeV. An overview of the bunch and machine parameters used for the measurements can be found in Table III. The first instability measurement determined the octupole current threshold in the absence of linear coupling, which is measured by slowly lowering the octupole current in steps until an instability develops. This allowed the characteristics of the instability to be acquired from the various diagnostic devices on the LHC [31]. This includes a headtail monitor, which enables the acquisition of the intrabunch motion, a beam synchrotron radiation telescope (BSRT) which measures the beam emittance from the emitted synchrotron light and the turn-by-turn base-band tune measurement device (BBQ), from which one can observe a bunch undergoing coherent oscillations as well as performing frequency domain analyses to acquire the tune and the azimuthal mode during the instability.

The second measurement took a bunch with similar parameters, introduced linear coupling (which allowed a measurement of the $\left|C^{-}\right|$via a tune crossing), and then slowly moved the tunes closer together in the presence of high octupole currents. The tune separation at which an instability is predicted to occur due to effects from linear coupling can then be calculated using the measured parameters.

In order to be able to make comparisons between different sets of measurements with small variations in beam parameters, each octupole threshold measurement is linearly scaled to a bunch with fixed parameters, here (for simplicity later) it is normalized to $N_{b}=1.2 \times 10^{11} \mathrm{p}$ and $\varepsilon=1.5 \mu \mathrm{m}$.

The single bunch measurement without linear coupling became unstable horizontally with $\left(Q_{x}^{\prime}, Q_{y}^{\prime}\right)=(9,8)$ and a damping time of $\tau_{d} \approx 100$ turns at an octupole current of $J_{\text {oct,th, } \mathrm{x}}=101 \mathrm{~A}$ [32]. An azimuthal mode 0 and 2 nodes in the headtail pattern were observed. The rise time of the instability was $\tau \approx 32 \mathrm{~s}$.
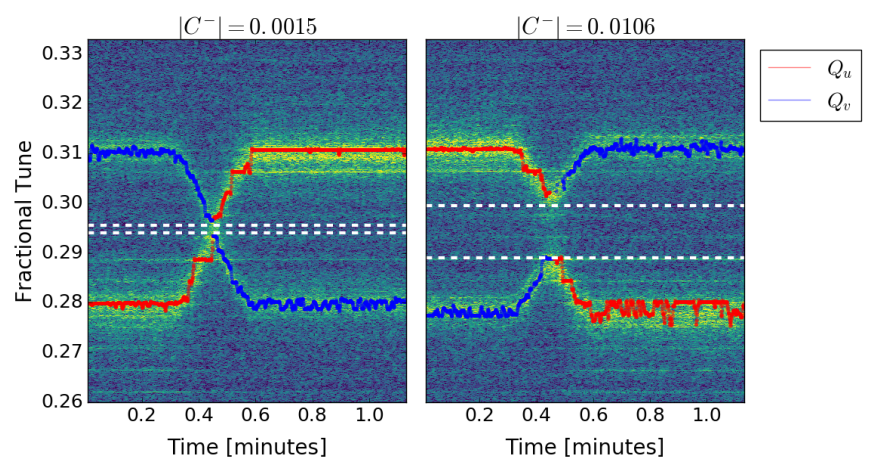

FIG. 9. Horizontal (red) and vertical (blue) fractional tunes as a function of time for each of the $\left|C^{-}\right|$measurements. The left plot was a measurement of the machine with well corrected coupling, and the right plot shows the coupling after the knobs were varied. The white dashed lines are used to compute the closest tune approach.

Immediately after this measurement, another single bunch was accelerated to $6.5 \mathrm{TeV}$ with the same machine parameters. The intention of the dedicated measurements was to introduce large coupling at the position of the LOs, and incite an instability with large LO currents present by moving the tunes closer together.

Available in the LHC control room are knobs to vary the real and imaginary components for the $f_{1001}$ resonance driving term at the location of the tune measurement pickups. In the case where the coupling is well corrected, the local variation in coupling is large. However when the knobs are varied to increase the global coupling, it has the effect of increasing the local coupling everywhere in the ring, which reduces the impact of local variations. In this particular regime, the $\left|C^{-}\right|$becomes an adequate parameter to describe the level of coupling at the LOs.

In the LHC there are two families of LO, one adjacent to a focusing quadrupole (LOF) and one adjacent to a defocusing quadrupole (LOD), despite the opposite currents, the final output strength of the octupole is the same.

First, the $\left|C^{-}\right|$was measured by performing a crossing of the horizontal and vertical tunes. The minimum tune separation in this case was measured to be $\left|C^{-}\right|=0.0015$. The coupling knobs were empirically varied to increase the $\left|C^{-}\right|$(based on previous optics measurements), and the tunes were then crossed again to provide the new measurement, which was $\left|C^{-}\right|=0.0106$. These measurements can be found in Fig. 9. The bunch did not go unstable during the second tune crossing because the crossing occurred very rapidly. Typically for the machine and bunch parameters used, the instabilities have rise times on the order of approximately $20 \mathrm{~s}$, whereas the crossing was completed in less than $6 \mathrm{~s}$.

Once coupling had been introduced, the tunes were slowly moved closer together with a constant current in the LOs of $J_{\text {oct }}=283$ A. An instability developed in the horizontal plane with $Q_{\text {sep,coupled }}=0.0191$, which with a 

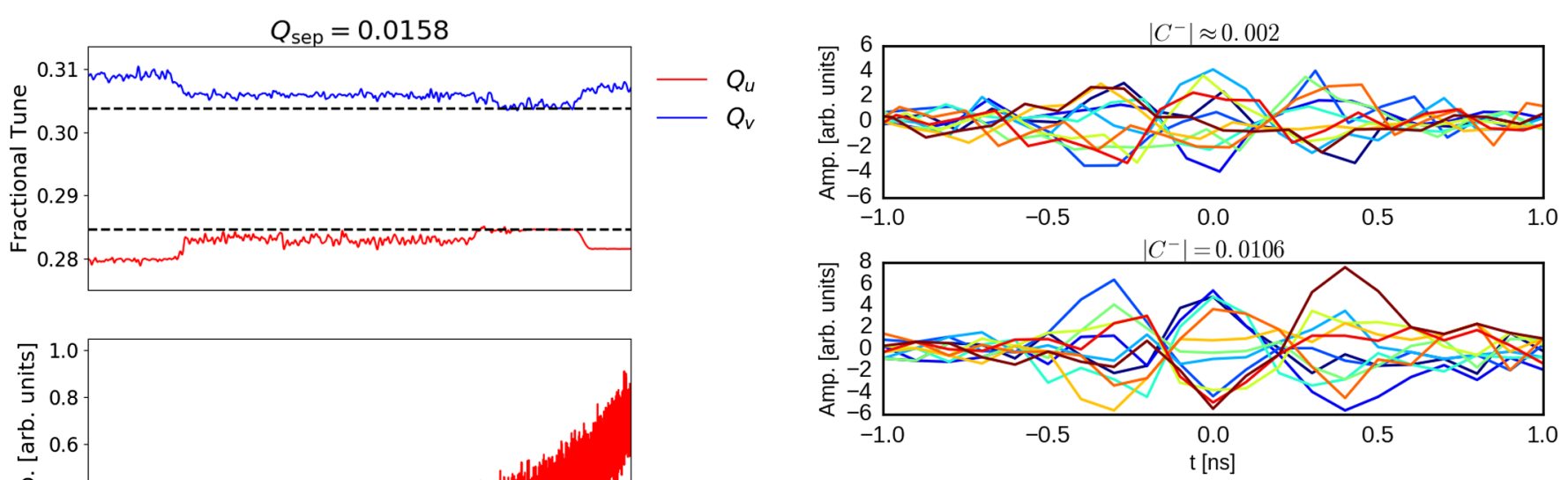

FIG. 11. Headtail monitor acquisitions during an instability without coupling (top) compared to the instability observed with coupling (bottom). 2 nodes in the headtail motion can clearly be seen in both cases. For both sets of measurements, $\left(Q_{x}^{\prime}, Q_{y}^{\prime}\right)=$ $(9,9)$ with the transverse feedback operating in nominal conditions.

FIG. 10. Top: Horizontal and vertical tunes as a function of time. Bottom: Horizontal BBQ amplitude vs time. An instability developed despite $J_{\text {oct }}=283 \mathrm{~A}$ in the LOs. The tune separation was calculated using the measured $\left|C^{-}\right|$.

$\left|C^{-}\right|=0.0106$ corresponds to $Q_{\text {sep }}=0.0158$. This is shown in Fig. 10.

The uncoupled normalized single bunch instability threshold had previously been measured as $J_{\text {oct,th }}=101 \mathrm{~A}$ in the horizontal plane, giving a factor increase in the required octupole current of 2.8 for these machine settings. Typically, the emittance measurement from the BSRT can have a $10 \%$ error bar attached to it, which is the dominant source of error out of all the bunch and machine parameters. This error can be propagated through to give a measured increase of $2.8 \pm 0.3$.

The headtail monitor was able to capture the intrabunch motion during the instability and can be seen in Fig. 11. The top plot shows the intrabunch pattern from the instability without coupling, and the bottom plot shows the instability incited by coupling. Both traces clearly show 2 nodes in the headtail motion. Along with the azimuthal mode 0 observed from this coupled headtail instability, it is clear that this instability is consistent with the uncoupled observations made in the previous measurement. This further confirms the assumption that in the event that both planes have similar characteristics (impedance, chromaticity, transverse feedback) then linear coupling does not impact on the coherent mode and only affects the tune footprint and thus the Landau damping.

A similar analysis to that found in Sec. III can be performed, but this time the tunes can be matched such that they are constantly separated by the measured coupled tune separation of $Q_{\text {sep,coupled }}=0.0191$. A frequency domain scan of the linear coupling and the LO current was performed using PYSSD and the results can be found in Fig. 12. The white dashed vertical line marks the measured

$\left|C^{-}\right|$which reaches a coherent stability factor of 1 (marking the threshold) at the point when the required current is 2.91 times more than for the uncoupled case.

The predicted increase in the octupole threshold was 2.91 , the measured increase in the octupole threshold was $2.8 \pm 0.3$, showing good agreement between the two approaches.

This measurement shows that if the tune separation is not maintained in the presence of large linear coupling and small coupled tune separations, Landau damping can be lost even in the presence of a large spread from LOs. The observations were in good agreement with simulations, which show that the characteristics of the unstable mode do not change in the event that both planes have similar properties.

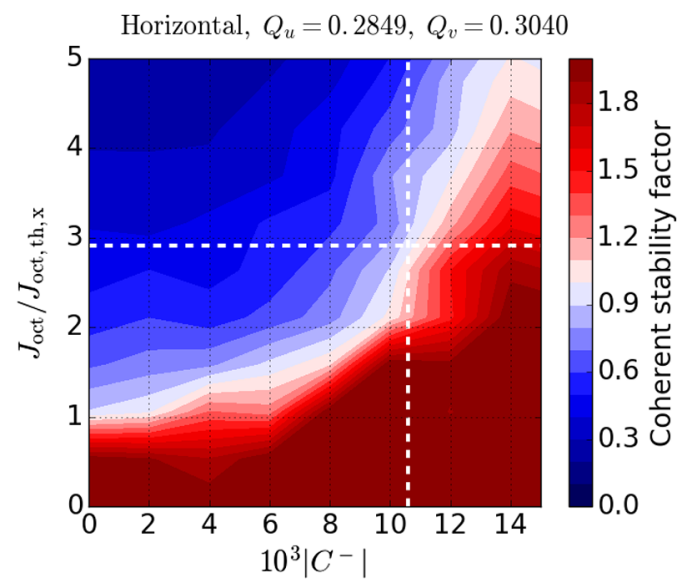

FIG. 12. PySSD results for a fixed coupled tune separation of $Q_{\text {sep,coupled }}=0.0191$. The white dashed vertical line marks the measured $\left|C^{-}\right|$which corresponds to an increase in the octupole threshold by a factor of 2.91 . 


\section{B. Uncontrolled}

There have been several cases in 2016 and 2017 when instabilities were observed during physics operation that could be related to linear coupling.

It has been seen that during collisions (when almost all of the approximately 2500 bunches have significant beambeam effects) the working point with a more optimized dynamic aperture (DA) is found with a reduced tune separation. When moving the working point closer to the coupling resonance, the noncolliding bunches (that are used for more accurate tune measurements) are regularly seen to undergo coherent oscillations and suffer emittance blowup. This is because of the fact that the ratio of the linear coupling to the tune separation becomes large, despite the fact the linear coupling is kept small (on the order of $1-2 \times 10^{-3}$ ).

\section{APPLICATION TO THE LHC STABILITY MODEL}

The octupole current required for stabilization varies depending on the size of the complex coherent tune shift. Many different factors can impact the size of this shift, such as chromaticity, transverse feedback, or the beam intensity. A combination of the tune spread and the beam emittance is important for determining if the coherent mode can be Landau damped. Linear coupling must now also be included in the stability model in order to be able to make accurate predictions for stability thresholds during operation of the LHC.

Figure 13 shows the required stabilizing octupole current (both positive and negative) for the horizontal plane as a function of $Q^{\prime}$. For each step, the coupled tunes are kept at $Q_{u}=0.31, Q_{v}=0.32$ and a damping time of $\tau_{d}=50$ turns is used. The impedance model used is for

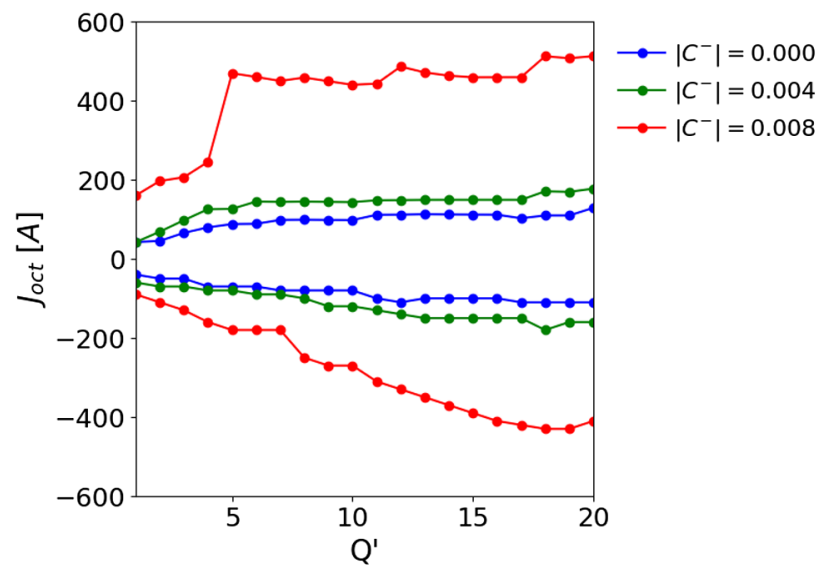

FIG. 13. Required stabilizing octupole current in the horizontal plane from PYSSD as a function of $Q^{\prime}$ for different values of $\left|C^{-}\right|$. This figure uses the LHC 2016 impedance model for an energy of $E=6.5 \mathrm{TeV}$. Constant tunes of $Q_{u}=0.31, Q_{v}=0.32$ were maintained for each point, with $\tau_{d}=50$ turns, $M=$ 2748 bunches and $\beta^{*}=40 \mathrm{~cm}$. a full machine (number of bunches $M=2748$ ) and $\beta^{*}=40 \mathrm{~cm}$. The three curves are for different values of $\left|C^{-}\right|$. It can be seen from the figure that for $\left|C^{-}\right|=0.004$, a small effect is found on the required stabilizing octupole current. However this becomes significant when the $\left|C^{-}\right|$ is increased. For $\left|C^{-}\right|=0.008$, a factor 3-4 times more octupole is needed to stabilize. For $J_{\text {oct }}<0$, the effect of coupling gets gradually worse with increasing chromaticity. This is because the tune spread is now in the opposite direction to that shown in Fig. 1 (with the spread opening out in the bottom left instead of the top right of the figure), making it easier to stabilize. For $J_{\text {oct }}>0$, large values that are up to the limit of the LHC octupoles $(550 \mathrm{~A})$ are required to stabilize.

It is clear that if efficient beam operation is to occur, the linear coupling needs to be kept small. The critical value is found when the $\left|C^{-}\right|$becomes approximately 50\%-60\% of the coupled tune separation. At this point, losing Landau damping is likely except in the presence of extremely high octupole currents. Linear coupling is now a critical part of the LHC stability model. To aid in the endeavour of keeping the effect of linear coupling on the beam dynamics as small as possible, an automatic correction of the Laslett tune shift is now applied during injection into the LHC, and new tools are being developed to better measure and correct the coupling in all stages of the machine cycle. If there are cases where the $\left|C^{-}\right|$is unable to be accurately controlled, then a large tune separation will be maintained to prevent instabilities.

It is worth noting that if the tune separation needs to be reduced to improve the DA and therefore the beam lifetime, then particular care needs to be paid to reduce the $\left|C^{-}\right|$.

\section{CONCLUSIONS}

Observations in the past, both at HERA and the LHC, have shown that if the linear coupling is high and the tune separation is small, then transverse instabilities can occur. Simulations have been performed with both particle tracking in the time domain and analytic computations in the frequency domain, which show that linear coupling can strongly perturb the tune spread acquired from the Landau octupoles. This can lead to transverse instabilities due to the loss of Landau damping. Dedicated measurements in the LHC verified the prediction from simulations. In machines that require Landau octupoles for stabilization, linear coupling must be considered when analyzing collective effects.

Linear coupling is now an integral part of the LHC stability model, and must be well corrected at all stages of the machine cycle. When the tune separation is small, the beam stability becomes dependent on the strength of the linear coupling. If the $\left|C^{-}\right|$becomes about $50 \%-60 \%$ of the coupled tune separation, the required Landau octupole current can increase by approximately a factor of 4 .

Some measurements from the LHC in 2012 have since shown that a $\left|C^{-}\right|$as high as 0.01 could have been present 
during some stages of the machine cycle. This highlights that linear coupling could have provided the missing factor 4 or 5 required for stability.

This study had focused on the effect of linear coupling on the tune spread generated by Landau octupoles only. The effect of linear coupling on other elements that generate a tune spread is currently under study for its potential impact on operation of the LHC. For example, there could be a strong dependence of linear coupling on the impact of beam-beam, space-charge, or electron cloud effects. Studying these mechanisms will allow a greater understanding of the machine and will continue to push the LHC to more challenging scenarios in the future.

\section{ACKNOWLEDGMENTS}

The authors would like to thank S. Fartoukh, E. H. Maclean, T. Persson and R. Tomás for helpful discussions on linear coupling and the LHC operations crew for their invaluable help during measurements.

\section{APPENDIX: LOCAL VS GLOBAL COUPLING}

Throughout this study, the $\left|C^{-}\right|$is used as a measure of the linear coupling in the machine. This is a global parameter of the ring, and is calculated by the minimum tune separation that can be achieved. However, this parameter does not take into account the local coupling variation throughout the lattice. In this Appendix, the modification of the tune footprint through linear coupling is disentangled between local and global coupling.

In order to investigate this, a coupling bump is introduced into one LHC sector (S12). By powering the octupoles only in one sector at a time, the effect on the tune footprint can be determined. To do this, 8 skew quadrupoles were artificially added in drift spaces in the LHC MAD-X lattice: 4 skew quadrupoles to the right of IP1, and 4 skew quadrupoles to the left of IP2. The strength of these 8 skew quadrupoles, together with the trim quadrupoles used to control the tune, were entered into a matching algorithm to introduce a local coupling bump in S12. The tunes were set to the design tunes used at injection energy $Q_{x}=0.28$, $Q_{y}=0.31$. The results of this matching can be found in Fig. 14. It can be seen that the coupling resonance driving terms (RDTs) only have an amplitude in S12, while being equal to 0 for the rest of the ring (this is not shown but can be inferred by there being no other skew quadrupoles powered and the RDTs being 0 either side of S12). For localized coupling to this sector, the global coupling is small $\left(\left|C^{-}\right|=0.0018\right)$ compared to the tune separation $\left(Q_{\text {sep }}=0.03\right)$.

The effect of this coupling bump on the tune footprint can now be calculated by comparing two cases: first by powering the octupoles in a different sector (S81) and comparing the footprints with and without the coupling bump in S12, and then by powering the octupoles in S12
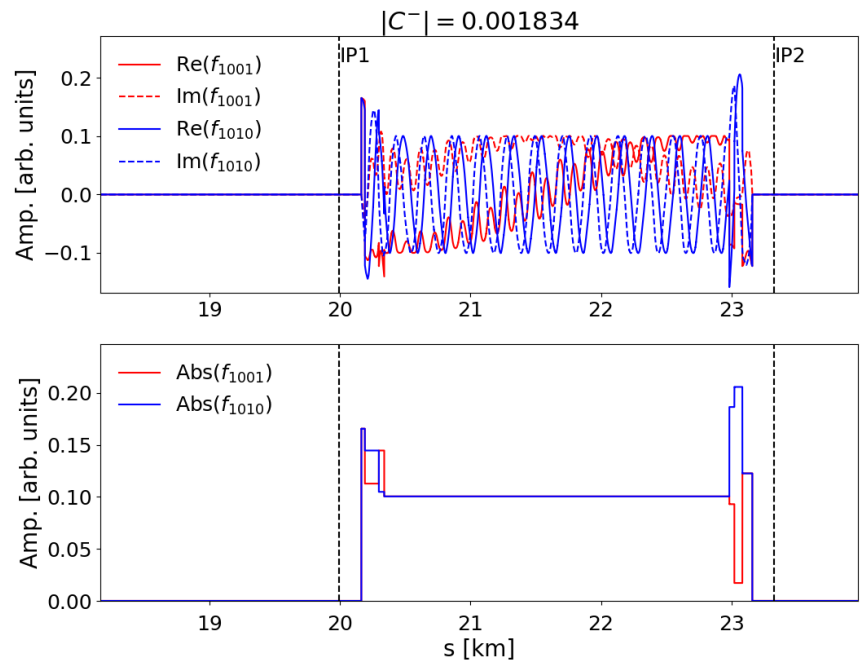

FIG. 14. Top: Real (solid) and imaginary (dashed) components for the coupling RDTs $f_{1001}$ (red) and $f_{1010}$ (blue) focused on Sector 12. Bottom: The absolute value of the RDT's in the same location. It can be seen that there is no coupling outside of this region, while maintaining strong coupling inside this region.

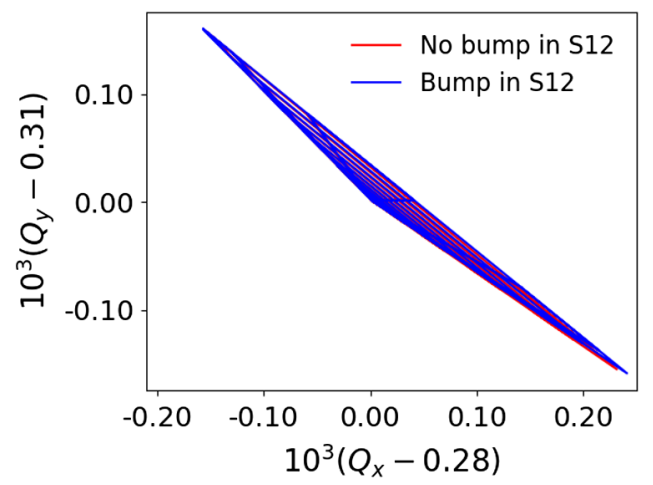

FIG. 15. Comparison of the tune footprints with LOs powered only in S81 and no coupling bump in S12 (red) or with coupling bump in S12 (blue). No impact on the footprint can be observed.

and comparing the footprint with and without the coupling bump (in the same sector).

Figure 15 shows the first case described above. All of the LOs are powered in S81 and the footprints are shown with and without the coupling bump in S12. It is clear to see that there is negligible difference between the two cases.

Figure 16 shows the second case described above, where the LOs are powered only in S12 and the footprints are compared between the cases of no coupling and with coupling bump. It can be seen that despite the small $\left|C^{-}\right|$and large $Q_{\text {sep }}$, a strong impact is observed on the tune footprint.

For both of these cases, the closest tune approach is kept constant at $\left|C^{-}\right|=0.0018$ and is small compared to the tune separation. This leads to the conclusion that when considering a real machine, local coupling at the point where a tune spread is created is the critical parameter for determining how strongly the footprint is modified. 


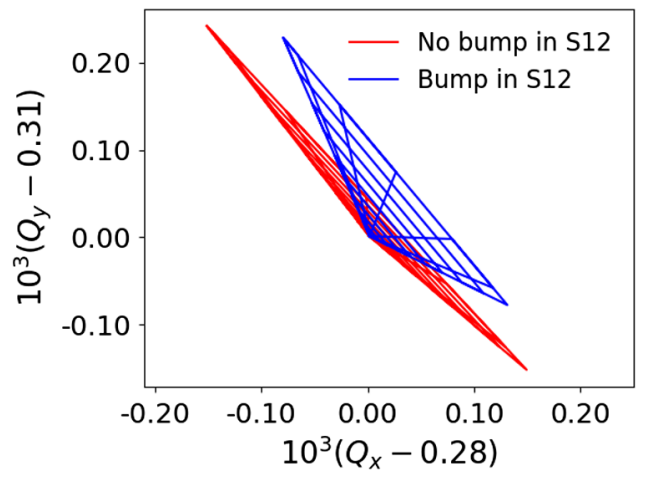

FIG. 16. Comparison of the tune footprints with LOs powered in S12 with the coupling bump (blue) and without the coupling bump (red) in S12. Despite the large tune separations and the small global coupling, the impact on the tune footprint is significant.

In a real machine, sources of local coupling are distributed throughout the ring and these sources are well corrected. However, that still means that in the case where there is a small global coupling, strong sources of local coupling could be present [for example in the interaction regions (IRs)], which could modify the footprint and therefore the Landau damping. From the point of view of the LOs, this has less of an effect as the octupoles are distributed throughout the arcs, but for beam-beam effects this could be significant.

In the LHC, when increasing the coupling using the skew quadrupoles in the arc cells, it has the effect of increasing the local coupling everywhere. This makes the impact from small local coupling bumps small and causes the coupling in the arcs to dominate. For simplicity in the simulation model, only the case with a single skew quadrupole is considered. In this case, as in the case where coupling is introduced in the real machine, the global coupling is directly related to the strength of the local coupling throughout the ring.

[1] E. Métral et al., Summary of the 2 day internal review of LHC performance limitations (linked to transverse collective effects) during run I, CERN Report No. CERN-ACCNOTE-2014-0006, 2014.

[2] L. R. Carver et al., Instabilities and beam induced heating in 2015, in Proceedings of the 6th Evian Workshop on LHC Operation, Evian, France (2015); Report No. CERNACC-2015-376, p. 95.

[3] A. Chao, Physics of Collective Beam Instabilities in High Energy Accelerators, 1st ed. Wiley Series in Beam Physics and Accelerator Technology (Wiley, New York, 1993).

[4] E. Métral, G. Hoffstaetter, and F. Willeke, Destabilising effect of linear coupling in the HERA proton ring, in Proceedings of the 8th European Particle Accelerator Conference, Paris, 2002 (EPS-IGA and CERN, Geneva, 2002), p. 1535.
[5] E. Métral, Theory of coupled Landau damping, Part. Accel. 62, 259 (1999); Report No. CERN-PS-99-011-CA.

[6] R. Cappi, E. Métral, and D. Möhl, Control of coherent instabilities by linear coupling, in 18th International Conference on High-energy Accelerators, Tsukuba, Japan (2001), p. P2new01.

[7] E. Métral, G. Rumolo, B. Salvant, and R. Steerenberg, Simulation study of the horizontal head-tail instability observed at injection of the CERN Proton Synchrotron, in Proceedings of the 22nd Particle Accelerator Conference, PAC-2007, Albuquerque, NM (IEEE, New York, 2007), p. 4210.

[8] E. Métral and G. Rumolo, Simulation study on the beneficial effect of linear coupling for the transverse mode-coupling instability in the CERN super proton synchrotron, in Proceedings of the 10th European Particle Accelerator Conference, Edinburgh, Scotland, 2006 (EPS-AG, Edinburgh, Scotland, 2006), paper THPCH058, p. 2916.

[9] A. Burov and V. Lebedev, Coherent motion with linear coupling, Phys. Rev. ST Accel. Beams 10, 044402 (2007).

[10] E. Maclean, F. Carlier, M. Giovannozzi, T. Persson, and R. Tomás, Effect of linear coupling on nonlinear observables at the LHC, presented at The 8th International Particle Accelerator Conference (IPAC'17), Copenhagen, Copenhagen, Denmark, May 2017, paper WEPIK092 (2017), pp 3151-3154.

[11] E. H. Maclean, R. Tomás, F. Schmidt, and T. Persson, Measurement of nonlinear observables in the Large Hadron Collider using kicked beams, Phys. Rev. ST Accel. Beams 17, 081002 (2014).

[12] S. White, E. H. Maclean, and R. Tomás, Direct amplitude detuning measurement with ac dipole, Phys. Rev. ST Accel. Beams 16, 071002 (2013).

[13] R. Tomás, T. Persson, and E. Maclean, Amplitude dependent closest tune approach, Phys. Rev. Accel. Beams 19, 071003 (2016).

[14] K. Li et al., Code development for collective effects, in 57th ICFA Advanced Beam Dynamics Workshop on HighIntensity, High Brightness and High Power Hadron Beams (HB2016), Malmö, Malmö, Sweden, July 2016, paper WEAM3X01 (2016), p. 362.

[15] E. Métral et al., Beam instabilities in hadron synchrotrons, IEEE Trans. Nucl. Sci. 63, 1001 (2016).

[16] https://github.com/PyCOMPLETE/PyHEADTAIL.

[17] N. Mounet, DELPHI: An analytic Vlasov solver for impedance-driven modes, in CERN BE-ABP-HSC Section Meeting, CERN, Geneva, Switzerland, 5th July 2014, https:// espace.cern.ch/be-dep-workspace/abp/HSC/Meetings/ DELPHI-expanded.pdf.

[18] X. Buffat, W. Herr, N. Mounet, T. Pieloni, and S. White, Stability diagrams of colliding beams in the Large Hadron Collider, Phys. Rev. ST Accel. Beams 17, 111002 (2014).

[19] http://cern.ch/madx.

[20] T. Persson and R. Tomás, Improved control of the betatron coupling in the Large Hadron Collider, Phys. Rev. ST Accel. Beams 17, 051004 (2014).

[21] E. Métral, Coupled landau damping of transverse coherent instabilities in particle accelerators, Ph.D. thesis, JosephFourier University, 1999. 
[22] A. Calia, K. Fuchsberger, M. Gabriel, G.-H. Hemelsoet, M. Hostettler, M. Hruska, D. Jacquet, M. Soderen, T. Persson, and D. Valuch, Online coupling measurement and correction throughout the LHC cycle, in 16th International Conference on Accelerator and Large Experimental Control Systems, Barcelona, Spain, ICALEPCS2017 (2017), p. 686.

[23] T. Persson, G. Baud, J. Coello de Portugal, M. Gasior, M. Giovannozzi, J. Olexa, R. Tomás, A. Garcia-Tabares Valdivieso, and D. Valuch, Linear coupling dependence on intensity and a next step towards a feedback (MD1850), CERN Report No. CERN-ACC-NOTE-2017-008, 2017.

[24] O. Brüning, P. Collier, S. Myers, R. Ostojic, J. Poole, and P. Proudlock, CERN Yellow Reports: Monographs (CERN, Geneva, 2004).

[25] D. Möhl and H. Schönauer, Landau damping by non-linear space-charge forces and octupoles, in Proceedings of the 9th International Conference on the High-Energy Accelerators (HEACC 1974): Stanford, CA (1974), p. 380.

[26] J. S. Berg and F. Ruggiero, Stability diagrams for landau damping, in Proceedings of the Particle Accelerator Conference, Vancouver, BC, Canada, 1997 (IEEE, New York, 1997), paper C970512, p. 1712.
[27] R. Bartolini and F. Schmidt, SUSSIX: A computer code for frequency analysis of nonlinear betatron motion, CERN Report No. CERN SL/Note 98-017 (AP), 1998.

[28] J. Gareyte, J.P. Koutchouk, and F. Ruggiero, Landau damping, dynamic aperture and octupoles in the LHC, LHC Project Report 91, Large Hadron Collider Project, 1997.

[29] N. Mounet, The LHC transverse coupled-bunch instability, Ph.D. thesis No. 5305, Ecole Polytechnique Federale De Lausanne, Lausanne, 2012.

[30] L. R. Carver et al., Current status of instability threshold measurements in the LHC at $6.5 \mathrm{TeV}$, in 7 th International Particle Accelerator Conference (IPAC16), Busan, Busan, Korea, May 2016, paper TUPMW011 (2016), p. 1434.

[31] T. Levens et al., Instability diagnostics, in Proceedings of the 6th Evian Workshop on LHC Operation, Evian, France, December (2015), p. 137; Report No. CERNACC-2015-376.

[32] L. R. Carver et al., MD1228: Validation of single bunch stability threshold \& MD1751: Instability studies with a single beam, CERN Report No. CERN-ACC-NOTE-20170013, 2017. 